\title{
Mechanism studies on the cellular internalization of nanoparticles using computer simulations: A review
}

\author{
Yun Hao Feng ${ }^{1}$, Bo Zhi Chen ${ }^{1}$, Wen Min Fei ${ }^{2}$, Yong Cui ${ }^{2}$, CAN YANG ZHANG ${ }^{3}$, and Xin \\ Dong Guo ${ }^{1}$ \\ ${ }^{1}$ Beijing University of Chemical Technology \\ ${ }^{2}$ China-Japan Friendship Hospital \\ ${ }^{3}$ Tsinghua Shenzhen International Graduate School
}

June 28, 2021

\begin{abstract}
Nanomaterial drug delivery systems have become one of the most important targeted therapy technologies. Although great efforts have been made to study the self-assembled mesoscopic structure of nanoparticles and understand drug loading and release mechanisms, the interaction between nanoparticles and cell membranes has not yet been clearly studied. Moreover, the research of experimental methods in this field has been greatly restricted due to its special time-space scale, so it is necessary to apply computer simulations to visualize the cell internalization of the nanoparticle. This review covers modelling methods and the current status and viewpoints of research on the influencing factors of the nanoparticle-biomembrane interaction mechanism. In particular, we discussed in detail the positive and negative effects of various nanoparticle properties. This article may assist researchers in rationally optimize the nanoparticle structure to improve therapeutic efficiency.
\end{abstract}

Mechanism studies on the cellular internalization of nanoparticles using computer simulations: A review

Yun Hao Feng, Bo Zhi Chen, Wen Min Fei, Yong Cui, Can Yang Zhang, Xin Dong Guo*

Beijing Laboratory of Biomedical Materials, College of Materials Science and Engineering, Beijing University of Chemical Technology, Beijing, 100029, P.R. China.

Key Lab of Biomedical Materials of Natural Macromolecules (Beijing University of Chemical Technology), Ministry of Education, Beijing 100029 China.

Corresponding author:

Xin Dong Guo, Beijing Laboratory of Biomedical Materials, College of Materials

Science and Engineering, Beijing University of Chemical Technology, Beijing,

100029, China. E-mail: xdguo@buct.edu.cn.

\begin{abstract}
Nanomaterial drug delivery systems have become one of the most important targeted therapy technologies. Although great efforts have been made to study the self-assembled mesoscopic structure of nanoparticles and understand drug loading and release mechanisms, the interaction between nanoparticles and cell membranes has not yet been clearly studied. Moreover, the research of experimental methods in this field has been
\end{abstract}


greatly restricted due to its special time-space scale, so it is necessary to apply computer simulations to visualize the cell internalization of the nanoparticle. This review covers modelling methods and the current status and viewpoints of research on the influencing factors of the nanoparticle-biomembrane interaction mechanism. In particular, we discussed in detail the positive and negative effects of various nanoparticle properties. This article may assist researchers in rationally optimize the nanoparticle structure to improve therapeutic efficiency.

Key words: computer modelling, dissipative particle dynamics, nanoparticle, cell membrane, internalization efficiency

\section{Introduction}

Since the 21st century, the concept of nanomedicine technology has been widely accepted ${ }^{1}$. Traditional drugs, especially cancer treatment drugs, often have poor water solubility, low bioavailability, and poor targeting, making it impossible to complete timely and effective drug treatment ${ }^{2}$. With the vigorous development of nanotechnology, the way of drug delivery has undergone earth-shaking changes. On the one hand, people have developed nano-micro tools to assist drug absorption through nanotechnology, such as polymer MNs, enhancing transdermal drug delivery efficiency by opening skin channels ${ }^{3}$. On the other hand, researchers have prepared a series of drug-loaded nanoparticles using nano chemical methods, which can wrap poorly water-soluble drugs inside the micelles, protected by the hydrophilic molecular fragments the outside of the micelles, thereby enhancing the drug's water solubility ${ }^{4}$. Those nanoparticles can also contain environmentally responsive components to adjust molecular properties according to changes in the external environment, by which the smart release of drugs can be obtained ${ }^{5}$. From now on, individuals have designed a large number of smart nanoparticles, which can effectively improve the treatment efficiency of anticancer drugs such as doxorubicin ${ }^{6}$ and paclitaxel ${ }^{7}$. Many drug-loaded particles can carry more functional components, having excellent development potential in medical imaging and sensing. The optimization design of more sensitive, versatile, and higher drug-loading nanosystems and even nanorobots' research and development have always been hot topics in this field ${ }^{8}$.

However, similar to other drug delivery systems, nanoparticle development also faces various challenges due to the diverse components and complex in vivo environment ${ }^{9}$. Hu et al. have used computer simulation to explore the influence of molecular structure on the self-assembly morphology of the drug carrier and summarized the formation mechanism of various self-assembly structures such as micelles, vesicles, and membranes ${ }^{10}$. Guo et al. have utilized molecular simulation technology to explore the drug release mechanism of $\mathrm{pH}$-responsive micelles, assisting researchers in the optimization design of nanomaterials ${ }^{11}$. Qiu et al. have put forward a variety of measures such as "physical cross-linking" to prevent drugs from leaking from the vesicles through experimental means ${ }^{12}$. Granick and his coworkers have studied the law of nanoparticle diffusion in polymer solution, which is beneficial for in-depth discussion of the nanoparticle delivery kinetics in the body ${ }^{13}$. At present, employing experiments and theoretical simulations, breakthroughs have been made in multiple aspects, such as drug loading efficiency, drug release mechanism, and nanoparticle dynamics research. However, most nanoparticles need to enter the cell in order to accomplish the delivery ${ }^{14}$. In the meantime, cellular cytotoxicity, the adverse effects of nanoparticles and macromolecules on cells, is also essential ${ }^{15}$. Therefore, it is of great pivotal to study the interaction between nanoparticles and cell membranes. Despite plenty of research has been reported in the fields of analyzing the nanoparticle cytotoxicity and the cell membrane morphology study by means of experimental methods ${ }^{16}$, there are still many areas where experiments cannot provide more complete support, including the absorption process as well as the interaction mechanism study at the molecular level. Thus, theoretical simulation plays a critical role in this topic. Through computer simulation, people can visualize nanoparticles' endocytosis process and study various influencing factors that affect the absorption efficiency by rich modelling methods, assisting researchers to project better nanoparticle designs that can improve internalization efficiency and avoid cell damage ${ }^{17}$. 
In this review, we will systematically review nanoparticle-cell membrane interaction research through computer simulation in recent years. Computer simulation refers to applying computer software to make reasonable assumptions in the movement of electrons and atoms ${ }^{18}$. At present, a rich type of molecular simulation technology has been developed that can be applied to the research of different space-time physics backgrounds. Considering the multiple components and the corresponding unique time-space scales, this review will focus on the application of dissipative particle dynamics (DPD) simulations based on the concept of coarse-grained in this field and several works that relate to the MARTINI force field are also mentioned ${ }^{11}$. First, we will review the commonly used modelling methods of nanoparticles and cell membranes. Subsequently, we will focus on reviewing many influencing factors that affect the interaction between nanoparticles and cell membranes, such as particle size, shape, chemical properties, concentration, etc., and summarize significant adjustment to improve the internalization efficiency of nanoparticles and the corresponding mechanism studies (Figure. 1). We hope this article may help molecular simulation researchers quickly familiarize themselves with the development of this field and various views on different researchers' mechanism research. At the end of the article, the author's comments and prospects will be given.

\section{Simulation Methodology}

\subsection{Modelling method of nanoparticle}

Rigid particle is the most frequently introduced model (Figure. 2A). This coarse-grained morphology is generally mapped from an existing particle model such as the Fcc lattice structure ${ }^{19}$. The coarse-grained beads are not necessary to be connected by bonds, but the relative position between the beads is usually required to be invariable to avoid shape degradation or even disintegration during the simulation process. In order to ensure the rigidity of nanoparticles, it is usually indispensable to furnish geometric constraints to the beads by adjusting the parameters of the potential energy equation:

where the eq (1) (3) is applied to bond length, bond angle, and dihedral angle, respectively. Based on different research purposes, the bead types on the particle surface can be changed or just be connected with other bead fragments to finish surface modifying. During this process, researchers can adjust the modification ratio and distribution according to actual needs to obtain a wide variety of nanoparticles. This particle modelling is very common in this field since its method is simple and can simulate some rigid inorganic particles and cell membranes. However, it cannot well reflect the mechanical behaviour of soft matter particles due to the neglect of the nanoparticle deformation. Another common nanoparticle model comes from most studies block copolymer self-assembly ${ }^{20-22}$. This type of particle contains a hydrophobic drug-carrier inside and a protective hydrophilic layer outside (Figure. 2B). This type of model conforms to the practical design of general amphiphilic polymer drug-loading nanoparticles. However, due to the flexibility of the particles, the nanoparticles will experience large deformations during the simulation process, which will lead to large energy fluctuations. Simultaneously, the insufficient cohesive energy may render nanoparticles disintegrate before the process of nanoparticles entering the cell to be observed ${ }^{23}$.

The complex components have also brought huge challenges to parameter controlling, so this modelling method is not commonly used in this field. To overcome this adversity, Pivkin ${ }^{24}$ et al. proposed a new method using a mesh model to model nanoparticles: each bead on the surface of the nanoparticle is a vertex of a two-dimensional curved triangular surface, and each vertex is connected by springs with a certain number of other vertices (Figure. 2C). The typical feature of this model is that it can adjust the elastic modulus of nanoparticles by altering many limited parameters to assist researchers in discussing the influence of nanoparticle elastic deformation on the process of cell absorption. Readers may check these references ${ }^{25,26}$ for the detailed control methods parameters.

In addition to building a nanoparticle model, the modeler should also consider the driving force for the nanoparticle to enter the cell, such as ligand-receptor binding, van der Waals force, hydrophobic interaction, chemical potential gradient, etc ${ }^{27}$. One simple method is to directly apply a spring force directed to the cell 
membrane on the nanoparticles ${ }^{28}$. The most common method is to define ligand beads on the surface of the nanoparticle and receptor beads on the surface of the cell membrane model, and the strong interaction between receptors and ligands can be defined by adjusting the force filed parameters between the two beads $^{29}$. For example, some researchers may set the repulsive parameters in the DPD force field of the two beads to approximately zero to highlight the strong attraction ${ }^{30}$. A more general method is to quantify the ligand-receptor interaction by Lennard-Jones (L-J) potential as follows ${ }^{31}$ :

among which, $\epsilon$ represents the strength of the ligand-receptor interaction. Since the background of this type of research often involves the cancer cell membrane where the negative charge density on the surface is significantly greater than that of normal cells ${ }^{23}$, researchers should also pay attention to the calculation of the electrostatic force. The long-range electrostatic forces in molecular dynamics simulation are generally handled by the Ewald summation or particle grid Ewald method. Furthermore, there are also many wellmatched electrostatic force models for specific simulation methods, such as the electrostatic force model for DPD force fields based on particle-particle-particle-mesh (PPPM) method proposed by Groot ${ }^{32,33}$. In short, the modelling of nanoparticles needs to consider various settings such as particle geometric constraints, surface modification and driving force.

\subsection{Modelling method of cell membrane}

The cell membrane is usually represented by the phospholipid bimolecular membrane model, involving two main aspects: the coarse-grained model of the phospholipid and the construction of bilayer topology. Figure 3A illustrates several common phospholipid coarse-grained models. The Y-shape phospholipid coarse-grained model, which contains three hydrophilic beads and two hydrophobic bead tails, is established by Groot ${ }^{34}$ and revised by Kranenburg ${ }^{35}$. Then, Shillcock and Lipowsky have proposed a $\lambda$-shape model that could better match the actual phospholipid structure ${ }^{36}$. These models emphasize the mapping of three heavy atoms into a coarse-grained bead, but this is not consistent with the conception of the MARTINI force field. Therefore, $\mathrm{Gao}^{37}$ et al. have established the H-shape model on the basis of the four-to-one scheme with more detailed bead classification according to the MARTINI force field and calculated the repulsive parameters suitable for the explicit / implicit-solvent DPD simulation ${ }^{38}$. They have also verified that this new force field could well reproduce the structure and thermodynamic properties of the bimolecular membranes ${ }^{37}$. Although the above model can more accurately reflect the real physical and chemical properties of cell membranes, the complicated bead classification and irregular topology have caused a great challenge for subsequent simulations. Therefore, more researchers have adopted more simplified models or even linear models to simulate the phospholipid molecule. Such a simplified model can greatly improve simulation efficiency, thereby broadening the scope of biomedical application.

It is inevitable that the simplified model may ignore many details of the cell membrane, so a huge amount of effort is obliged to ensure that the formed bimolecular membrane conforms to the morphological and mechanical properties of the real cell membrane. First, a weaker bond length constraint is supposed to be given to the first hydrophobic bead of the two hydrophobic tails in the lipid model to ensure that the majority of phospholipid molecules can maintain a reasonable phospholipid tail orientation ${ }^{39}$. Simultaneously, the bond angle constraint is also applied to ensure the phospholipid molecule's rigidity ${ }^{31}$. Besides, the boundary conditions may cause the molecular relaxation, thereby destroy the continuity of the membrane. In order to reduce this effect, it is necessary to maintain the global conformation of the molecular membrane by limiting the movement in the longitudinal direction of the phospholipids near the boundary region ${ }^{27}$.

Furthermore, the N-varied DPD method is introduced to control the membrane tension, which can monitor the number of phospholipids per unit area (LNPA) in the boundary area. When LNPA is lower than the set value (the value of the tensionless membrane equals $0.64 \mathrm{~nm}$ ), a certain number of water beads in this area will be replaced with phospholipid beads to help researchers maintain membrane tension or artificially increase external tension ${ }^{40}$. Figure $3 \mathrm{~B}$ is a common coarse-grained phospholipid bimolecular membrane model. Generally, the rationality of the model is manifested by the membrane thickness and the phospholipid diffusion coefficient which should be close to $8 \mathrm{~nm}$ and $5 \mu \mathrm{m}^{2} \mathrm{~s}^{-1}$, respectively ${ }^{41}$. 
There are multiple components in cell membranes and diverse types of phospholipids. Beena Rai's group ${ }^{19}$ has used the MARTINI force field to construct a multi-component cell membrane containing different phospholipid molecules and embedded transport proteins as shown in Figure 3C. This model has been utilized to study the interaction between nanoparticles with different shapes as well as chemical properties and cell membranes. Yang ${ }^{42}$ et al. have discussed the influence of phase separation of disordered-gel phase on nanoparticle interaction by introducing unsaturated phospholipid. With the development of abundant modelling methods for nanoparticles and cell membranes, the credibility of computational simulation results has also been greatly enhanced.

\section{Parameters determining the NP-CM interaction}

\subsection{Nanoparticle Size}

It is intuitive to believe that the difficulty for nanoparticles entering the cell membrane will raise with the increasing particle size. Essentially, many researchers have also found that some nanomaterials with greater size will cause more critical changes in the morphology and surface composition of cell membranes. Jirasak ${ }^{43}$ et al. have found that small-sized nanoparticles similar to fullerenes can quickly enter the hydrophobic region of the lipid-bilayer without causing significant cell membrane deformation. Similarly, Rai Beena ${ }^{44}$ et al. have used a rigid nanoparticle model to measure the impact of the size effect of nanoparticles on cell membranes by analyzing some structure factor of membrane plane projection area, area compressibility, and order parameters. It has been found that larger-sized nanoparticle will obviously cause changes in the morphology and physical properties of the cell membrane (Figure. 4A). In addition, it was also found that small graphene sheets can quickly enter the inside of the bilayer, while large graphene sheets can only be partially embedded in the cell membrane and lead to more complex cell membrane deformations ${ }^{45}$. For graphene sheets adsorbed on the cell membrane surface, the orientation of lipid molecules in the area covered by the graphene sheets alters significantly driven by the hydrophobic interaction, and there is the phenomenon of more obvious the horizontal orientation of the lipid hydrophobic chains to be discovered with enlarging the size of graphene sheet model $^{46}$.

However, recent studies have shown that the size effect and the difficulty of entering the cell membrane are not a simple linear relationship, which indicates that there is theoretically a minimum and an optimal radius for entering the cell membrane ${ }^{47,48}$. As the radius is lower than the minimum radius, the cell membrane cannot be endocytosed, entering the cell only through direct penetration ${ }^{49}$. Meanwhile, for nanoparticles smaller than the optimal radius, large-sized particles can be more easily wrapped by cell membranes than small-sized particles (Figure. 4B) ${ }^{50}$.

The existence of the minimum radius lies in the competition of the adsorption energy and the cell membrane bending energy ${ }^{47}$. For larger nanoparticles, the number of receptors on the surface will be relatively higher, resulting in higher adsorption energy. Simultaneously, to wrap large-size nanoparticles, the curvature of the cell membrane's bending deformation should be larger, the membrane bending energy barrier, which is negatively related to the bending curvature ${ }^{51}$, would also be lower. On the contrary, nanoparticles of too small size cannot provide sufficient binding sites and small curvature, resulting in lower adsorption energy and greater membrane bending energy, rendering it more difficult to be wrapped by cell membranes. Therefore, only nanoparticles larger than the critical size can be endocytosed by the cell membrane. Gao ${ }^{47,50}$ et al. have also proposed another pair of competing interactions, the diffusion kinetics of nanoparticles near the cell membrane and the nanoparticle-cell membrane's thermodynamic attraction. Larger size nanoparticles may carry a larger number of ligands, and their diffusion performance is weaker due to their size effect, so they are more likely to be wrapped by cell membranes. In comparison, the higher diffusion coefficient of small-sized particles in the solution may give rise to an adverse effect on entering the cell membrane. However, for nanoparticles with an excessively large size, the wrapping time will increase significantly ${ }^{52}$, and the cytotoxicity caused by larger deformations will also affect the nanoparticles' therapeutic efficiency, so 
there is supposed to exist an optimal wrapping radius for the nanoparticles. To determine the optimal radius, some researchers have already begun to put forward insights on this issue from the perspective of the diffusion dynamics of nanoparticles. Yan ${ }^{53}$ et al. have studied the diffusion kinetics of graphene in cell membranes. They have pointed out that the diffusion kinetics of graphene sheets in cell membranes are affected by graphite interaction with hydrophobic phospholipid tails. Several typical graphene diffusion dynamics models have also been summarized: Brown dynamics-Levy dynamics-directional dynamics. Many studies have established a variety of mathematical models for the diffusion of nanoparticles, and their influencing factors are also be analyzing, which would assist people to find the optimal nanoparticle radius ${ }^{54}$.

\subsection{Nanoparticle Shape}

Shape is also a significant factor in determining how nanoparticles enter cells. For nanoparticles with good isotropy, such as spherical and cubic nanoparticles, the way they enter the cell is determined by their size: small-sized nanoparticles will directly penetrate through the cell membrane ${ }^{31,55}$. In contrast, larger nanoparticles need to be wrapped by the cell membrane to accomplish cell internalization ${ }^{56}$. For non-isotropic materials, the researchers have found that, under a strong driving force (concentration gradient, ligandreceptor interaction, etc.), the sharp end of materials can also penetrate the cell membrane ${ }^{57}$. By adjusting the surface chemistry of such nanoparticles, the kinetic process of nanoparticles piercing the cell membrane can be optimized (detailed discussion will be carried out in the next section). For the process of endocytosis, anisotropic materials possess a particular dynamic process: orientation-wrapping-reorientation ${ }^{58}$. Their initial conformation plays an extremely important role for anisotropic materials, such as rod-shaped, discshaped, and elliptical nanoparticles. Taking rod-shaped particles as an example, if the particles' initial conformation is perpendicular to the cell membrane, direct wrapping needs to overcome a large amount of membrane bending energy. Therefore, the nanoparticle will preferentially adjust its original orientation so that its morphological long axis could be parallel to the cell membrane, resulting in the lower bending energy (Figure. 5A). After being wrapped by the cell membrane, some nanoparticles may continue to rotate due to the residual rotational momentum (Figure. 5B) ${ }^{19}$. As reported in the previous study, this special internalization kinetics will reduce the efficiency of nanoparticles entering cells. $\mathrm{Li}^{59}$ et al. have used DPD simulations to verify that spherical and cubic nanoparticles with better isotropy can be wrapped by cell membranes within simulation time of 7 - $9 \mathrm{~ns}$, while rod-like and disc-like particles with poor isotropy would not finished the internalization within 13.5 ns (Figure. 5C). However, this preferentially oriented dynamic behavior can help cell membranes abate the membrane bending energy needed to overcome in the process of the endocytosis ${ }^{59}$. Chen et al. have also used Langevin's molecular dynamics simulation to demonstrate that some deformable spherical nanoparticles will also deform into an asymmetric structure and then be wrapped by cell membranes (Figure. 5D) ${ }^{26}$.

From the above discussion, it can be seen that the study of the rotation dynamics of nanoparticles is very indispensable for understanding nanoparticle internalization. $\mathrm{Chen}^{60}$ and his colleagues have studied the diffusion mechanism of nanoparticles by analyzing the mean square displacement. It has been found that charged particles possess better diffusion ability than neutral particles. Simultaneously, the nanoparticles with randomly distributed electric charges conform to the law of super diffusion (the nonlinear power-law relationship between the mean square displacement and the simulation time ${ }^{61,62}$ ), while the diffusion of uniformly distributed nanoparticles is more in line with the law of linear diffusion. By analyzing the typical trajectory and turning angle distribution of nanoparticles, it has been found that nanoparticles with randomly distributed charges would be inclined to diffuse in a specific direction due to more complex asymmetric forces and torques to be given to nanoparticles with random charge distribution, while nanoparticles with uniform charge distribution have almost the same probability of diffusing in all directions. $\mathrm{Ji}^{63}$ et al. have also found that nanoparticles modified with more or shorter ligands are more likely to interact with cell membranes, since longer modifiers may destroy the anisotropy of the nanoparticles, leading to the inhibition of the nanoparticle rotation $^{59}$. However, the rotation of nanoparticles can also bring disadvantages. Both experiments and DPD simulations have indicated that the rotation of nanoparticles will damage the surface structure of the cell membrane ${ }^{64}$. Balancing the relationship between cytotoxicity caused by the rotation of nanoparticles and 
optimizing the energy barrier of cell internalization is the next essential research topic in this field.

\subsection{Chemical property of nanoparticle surface}

The surface chemistry of nanoparticles is supposed to be the most critical factor affecting the interaction with cell membranes. In this review, more emphasis would be laid on three aspects: hydrophilic / hydrophobic properties, charge properties, and ligand-receptor interaction. In every aspect, the density surface pattern and the covalent bonding of the modification would have an important influence.

The hydrophilic surface modification of nanoparticles is the basis for successful drug delivery. For one thing, hydrophobic nanoparticles would reduce the biocompatibility of the drug delivery system ${ }^{65,66}$. For another, under the protection of the hydrophilic modifier, the bioavailability of nanoparticles will be guaranteed by avoiding being adsorb to plasma proteins ${ }^{30}$. However, it can be seen from Figure. 6A that the hydrophilic surface also affects the interaction between nanoparticles and cell membranes. Driven by the hydrophobic attraction, the nanoparticles can be trapped into the cell membrane faster, while nanoparticles with hydrophilic surfaces can only adsorb on the phospholipid head layer of the cell membrane, requiring to overcome a higher free energy barrier due to the incompatibility between the nanoparticle and the phospholipid tail $^{67}$. Similarly, in the study of graphene and cell membranes, researchers have also found that graphene can form a sandwich shape with cell membranes, while graphene oxide can be adsorbed on the cell membrane surface or vertically pass through the cell membrane conformation ${ }^{46,68}$. Therefore, researchers should appropriately adjust the hydrophilic / hydrophobic ratio of the nanoparticle surface. Furthermore, many experimental and theoretical studies have indicated that the hydrophilic / hydrophobic surface pattern can also have bearing on the cell internalization ${ }^{69}$. Compared with Janus particles, the nanoparticles in which two kinds of surface patterns alternately distributed will increase the probability of entering the cell. The study of Wang and his colleagues ${ }^{25}$ has pointed out that the more alternate surface pattern stratification, the higher the efficiency of cell internalization. Zhang ${ }^{67}$ et al. have also proved that nanoparticles with randomly distributed hydrophilic / hydrophobic surfaces can penetrate cell membranes, while Janus particles can only be trapped in cell membranes. In addition to affecting the efficiency of cell internalization, the surface properties of nanoparticles will also change cell membranes' physical and chemical properties. On the one hand, passing through the cell membrane will cause the cell membrane deformation. On the other hand, $\operatorname{Lin}^{70}$ et al. have discovered that hydrophobic nanoparticles will not change the cell membrane's phase transition temperature. As the degree of surface modification strengthens, the phase transition temperature would first increase with the subsequent decrease, leaving the final phase transition temperature higher than the original value.

Compared with normal cells, the cell membrane of cancer cells contains a higher density of negative charges ${ }^{71}$. Hence, the charge modification of nanoparticles is also an effective alternative means to enhance drug delivery efficiency. For example, researchers have proved that nanoparticles modified with zwitterionic polymers are better capable of consummating the cell internalization ${ }^{72}$. It has been reported that different charge types determine distinct cell membrane deformation mechanisms: neutral nanoparticles will not significantly change the lateral curvature of the cell membrane. Positively charged nanoparticles would cause the cell membrane to bulge upwards, while negatively charged particles cause the cell membrane to dent downwards (Figure. $6 \mathrm{~B})^{60}$. Moreover, for $\mathrm{pH}$ amphoteric drug-loaded nanoparticles, the charge effect is particularly significant. As the degree of ionization increases, the charge effect can help improve the efficiency of nanoparticles penetrating through the cell membrane. However, nanoparticles would disintegrate before being internalized by the cell with too much mounting degrees of ionization ${ }^{23}$. Therefore, these simulation results suggest that people need to adjust the relationship between nanoparticle cohesion and ionization. Another major factor, manifested from several studies, lies in choosing the covalent bond and non-covalent bond modification ${ }^{73}$. Rigid nanoparticles covalently linked to the modified polymer containing ligands can be wrapped by the cell membrane more productively due to the strengthening connection between nanoparticles and modifiers. Adversely, non-covalently attached nanoparticles cannot be wrapped by the cell membrane as the modification polymer detaching from the nanoparticle. Under the $\mathrm{pH}$ trigger, the modification polymer connected 
by non-covalent bonds has a significant increase in hydrophilicity then falls apart from the surface of the nanoparticle so that the core of the hydrophobic nanoparticle has the opportunity to enter the middle layer of the cell membrane.

Another key chemical modification is the ligand-receptor distribution, which assists nanoparticles specifically recognize targeted cells without damaging normal cells. Only sufficient ligand density and ligand-receptor interaction strength can induce cell membrane deformation and encapsulate nanoparticles. Therefore, understanding the relationship between the strength of the nanoparticle ligand-receptor interaction and the membrane tension can provide more insights to understand the mechanism of nanoparticle-cell interaction. As shown in Figure. 6C, the stronger the ligand density $(f)$ on the surface of the nanoparticle, the greater extent of the cell membrane deformation, which results in more complete membrane wrapping. Nevertheless, the mounting cell membrane tension $(\sigma)$ increase the difficulty of the membrane deformation, the nanoparticles thus tend to penetrate the cell. Therefore, by adjusting the relationship between $f$ and $\sigma$, five nanoparticle-cell membrane interaction mechanisms can be observed: (1) Direct penetration (lower $f$ and higher $\sigma$ ). (2) Penetration or partial wrapping (lower $f$ and moderate $\sigma$ ). (3) Full wrapping (higher $f$ and lower $\sigma$ ). (4) Wrapping following penetration (higher $f$ and appropriate $\sigma$ ). (5) Wrapping- penetrationrewrapping (lower $f$ and lower $\sigma$ ).

\subsection{Nanoparticle concentration}

The concentration gradient is also one of the driving forces for the interaction between nanoparticles and cell membranes. Therefore, both experimental and theoretical studies have indicated that nanoparticle concentration has a huge impact on internalization efficiency ${ }^{43}$. Multiple nanoparticles will aggregate or disperse on the cell membrane surface, which adjusts the nanoparticles' size and shape to affect the nanoparticle-cell membrane interaction mechanism.

For example, high-concentration nanoparticles lead to larger aggregates. As described in section 3.1, large size aggregates can reduce the membrane bending energy, thereby promoting the cell membrane to encapsulate the nanoparticles. Sometimes, the aggregates of nanoparticles will rearrange and recombine into various shape to increase the required curvature of the cell membrane, improving the efficiency of nanoparticle cell internalization. $\mathrm{Hu}^{74}$ et al. have explored the effect of peptide concentration alteration, and it can be seen from the simulation results that peptides can be adsorbed on the surface of the cell membrane and can open perforations in the cell membrane to enter the cell as the concentration is high. Bundling multiple small peptides into large individuals will also accelerate the pore-forming effect, which is similar to the concentration effect.

Inspired by the concentration effect, the researchers have proposed a method of cooperative internalization of multiple nanoparticles ${ }^{75}$. As mentioned in section 3.3, hydrophilic nanoparticles can be adsorbed on the hydrophilic surface of the cell membrane, but it is challenging to enter the hydrophobic inner layer of the cell membrane. With the aid of a partner nanoparticle, the hydrophilic nanoparticle can smoothly penetrate cell membranes under their synergistic effect, and the difference in nanoparticle surface properties can produce different degrees of cooperative internalization (Figure. 7A). A single hydrophilic nanoparticle is adsorbed on the cell membrane surface, while four hydrophilic nanoparticles can penetrate the cell membrane together. A single nanoparticle with a random hydrophilic / hydrophobic surface distribution can penetrate the cell membrane but can only be embedded in the cell membrane after increasing the concentration. Zhang ${ }^{74}$ et al. have analyzed the force spectrum of nanoparticles and the distance between nanoparticles. They have concluded that nanoparticle synergistic effect is greatly relevant to the dispersion behaviour of the nanoparticles. The aggregation of nanoparticles is more conducive to penetration, which is related to the difference in force status of the nanoparticles on the horizontal plane. Although the influencing factors and mechanisms of synergistic interaction are given, there are still counter-intuitive aspects. Cite an instance, the diffusion performance of hydrophilic nanoparticles in an aqueous solution should be better than that of hydrophobic nanoparticles. However, according to their simulation results, the distance between hydrophilic nanoparticles is smaller than that of hydrophobic nanoparticles. $\mathrm{Li}^{76}$ et al. have also studied the influence of nanoparticle 
charge on the synergistic penetration effect. It has been found that two positively charged nanoparticles can still enter the cell membrane since the interaction between the nanoparticle and the cell membrane weakens the electrostatic repulsion. In addition to the nanoparticle surface properties, the difference in their shapes will also cause different synergistic effects. For example, vertically-oriented asymmetric nanoparticles can further promote cell membrane penetration ${ }^{28}$. In order to promote the cooperative internalization between the nanoparticles, many researchers would choose to use polymers to connect the two nanoparticles. According to Figure. 7B, two nanoparticles connected by a polymer can increase the degree of engulfment by the cell membrane under the synergistic effect. The chemical properties, length, and molecular rigidity of the polymer connecting the nanoparticles should be systematically optimized when designing such a system. If too long and rigid polymer molecular chain has been utilized, it will increase the distance between the nanoparticles and reduce the synergistic effect of the nanoparticles ${ }^{77}$.

\subsection{Nanoparticle elastic modulus}

Although most researchers use rigid nanoparticle models to simulate the cell internalization process, with the diversification of modelling methods, more emphasis has been paid to nanoparticle mechanical properties, especially the elastic modulus ${ }^{78}$. As illustrated in Figure 8A, rigid hydrophilic nanoparticles have a higher free energy barrier to penetrate the cell membrane due to the incompatibility between the hydrophilic nanoparticle surface and the interior of the lipid bimolecular membrane. After appropriately reducing the nanoparticle elastic modulus, the nanoparticles would undergo a certain degree of deformation, which reduces the contact area between the nanoparticles and the lipid tail. Therefore, a lower energy barrier is supposed to be overcome by the softer hydrophilic nanoparticles. On the contrary, since the good attractive interaction with the middle part of the cell membrane for rigid hydrophobic nanoparticles, the free energy will decrease during the process of the nanoparticle penetrating the cell, which reaches a minimum as the nanoparticle is trapped in the middle of the cell membrane. The deformation of the softer nanoparticles changes the contact state between the nanoparticles and the cell membrane, enlarging the minimum value of free energy, making it more arduous for the nanoparticles to penetrate the cell membrane ${ }^{25}$. For the process of endocytosis, the effect of elastic modulus is also twofold. For one thing, $\mathrm{Yi}^{79}$ et al. have used the framework of Helfrich theory to propose a theoretical model for the interaction between elastic nanoparticles and cell membranes. From the numerical simulation results, it can be found that soft nanoparticles are more challenging to be wrapped by cell membranes than rigid nanoparticles because the deformation of nanoparticles will bring more energy. For another, the nanoparticle deformation will adjust the nanoparticle shape. If the deformation leads to an appropriate shape, which needs enlarged cell membrane curvature, thereby improving the nanoparticle internalization efficiency (Figure. $8 \mathrm{~B})^{26}$. Excessively lower nanoparticle elastic modulus will bring more negative effects, such as low nanoparticle stability under the shear of fluid and even the disintegration before being internalized by cells ${ }^{80}$.

\section{Conclusions}

In general, the research on nanoparticle cell internalization mechanism is very beneficial to improve the therapeutic efficiency of nanomaterial drug delivery systems. Both the nanoparticle and the cell membrane contain diverse chemical components and complex physical properties. Therefore, the effect of the properties of nanoparticles and cell membranes on the interaction is the hot-spot in this field. This article reviews the research on the factors affecting the internalization mechanism of nanoparticle in recent years and summarizes the positive and negative influence of multiple physical and chemical properties of nanoparticles, which will assist researchers to propose more reasonable nanoparticle design solutions:

(1) Nanoparticle size. Small size nanoparticles can smoothly penetrate cell membranes without causing significant cell membrane deformation. However, it also brings a lower number of surface ligands and a higher cell membrane bending energy barrier, thus properly increasing the nanoparticle size can increase 
the internalization efficiency. Hence, researchers should balance the relationship between cytotoxicity and membrane bending energy to determine the optimal size of nanoparticles.

(2) Nanoparticle shape. The asymmetrical shape (rod, ellipse, disc, etc.) nanoparticles are conducive to the penetration of cell membranes and have a special endocytosis dynamic: orientation-wrapping-reorientation. Compared with the isotropic shape (spherical, etc.) nanoparticles, this exceptional internalization kinetics results in a longer internalization time, but its conformation can be optimized to reduce the curvature of the cell membrane due to the orientation process which can also promote the wrapping of cell membranes.

(3) Nanoparticle surface properties. Hydrophobic nanoparticles can be embedded in the middle layer of the cell membrane, while hydrophilic nanoparticles can only be adsorbed on the surface. Therefore, researchers should reasonably adjust the ratio and distribution of the hydrophilic / hydrophobic surface of the nanoparticles. The density, length, and type of ligands on the nanoparticle surface will also affect the nanoparticle-cell membrane interaction mechanism, thereby changing the degree of nanoparticle encapsulation by the cell membrane. Moreover, since cancer cells usually contain a higher negative charge density, the charge distribution on the nanoparticle surface will also provide a greater driving force.

(4) Nanoparticle concentration. A high concentration of nanoparticles can form aggregates, increasing the size and adjusting the shape of the aggregates so that the nanoparticles can enter the cell together under the synergistic effect of the partner nanoparticles. Understanding the nanoparticle diffusion kinetics can support researchers in understanding the deep-depth mechanism of concentration effects.

(5) Nanoparticle elastic modulus. The nanoparticles with low elastic modulus are more difficult to internalize by cells due to additional deformation energy. However, a suitable elastic modulus can allow nanoparticles to flexibly modify their shape, thereby enhancing the cell internalization probability.

\section{Data availability statement}

The data are available from the corresponding author on reasonable request.

\section{Acknowledgments}

This work was financially supported by the National Natural Science Foundation of China (51873015, 51673019), the Research projects on biomedical transformation of China-Japan Friendship Hospital (PYBZ1817), and the long-term subsidy mechanism from the Ministry of Finance and the Ministry of Education of PRC. The calculation in this work is supported by high performance computing platform of BUCT.

\section{References}

1. Xiang H, Lin H, Yu L, Chen Y. Hypoxia-irrelevant photonic thermodynamic cancer nanomedicine. ACS Nano.2019;13(2):2223-2235.

2. Tila D, Ghasemi S, Yazdani-Arazi SN, Ghanbarzadeh S. Functional liposomes in the cancer-targeted drug delivery. J. Biomater. Appl. 2015;30(1):3-16.

3. He Y, Hong C, Li J, Howard MT, Li Y, Turvey ME, Uppu DSSM, Martin JR, Zhang K, Irvine DJ, Hammond PT. Synthetic charge-invertible polymer for rapid and complete implantation of layer-by-layer microneedle drug films for enhanced transdermal vaccination. ACS Nano.2018;12(10):10272-10280.

4. Radha B, Senesi AJ, O'Brien MN, Wang MX, Auyeung E, Lee B, Mirkin CA. Reconstitutable nanoparticle superlattices. Nano Lett.2014;14(4):2162-2167. 
5. Zhang CY, Xiong D, Sun Y, Zhao B, Lin WJ, Zhang LJ. Self-assembled micelles based on pH-sensitive PAE-g-MPEG-cholesterol block copolymer for anticancer drug delivery. Int. J. Nanomed. 2014;9:4923-4933.

6. Yoo HS, Park TG. Folate receptor targeted biodegradable polymeric doxorubicin micelles. J. Controlled Release. 2004;96(2):273-283.

7. Guo XD, Tan JPK, Kim SH, Zhang LJ, Zhang Y, Hedrick JL, Yang YY, Qian Y. Computational studies on self-assembled paclitaxel structures: Templates for hierarchical block copolymer assemblies and sustained drug release. Biomaterials. 2009;30(33):6556-6563.

8. Hu Q, Li H, Wang L, Gu H, Fan C. DNA nanotechnology-enabled drug delivery systems. Chem. Rev. 2019;119(10):6459-6506.

9. Ha D-H, Islam MA, Robinson RD. Binder-free and carbon-free nanoparticle batteries: A method for nanoparticle electrodes without polymeric binders or carbon black. Nano Lett.2012;12(10):5122-5130.

10. Tan HN, Wang W, Yu CY, Zhou YF, Lu ZY, Yan DY. Dissipative particle dynamics simulation study on self-assembly of amphiphilic hyperbranched multiarm copolymers with different degrees of branching. Soft Matter. 2015;11(43):8460-8470.

11. Feng YH, Zhang XP, Zhao ZQ, Guo XD. Dissipative particle dynamics aided design of drug delivery systems: A review. Mol. Pharmaceutics. 2020;17(6):1778-1799.

12. Liang $\mathrm{L}, \mathrm{Fu} \mathrm{J}$, Qiu L. Design of $\mathrm{pH}$-sensitive nanovesicles via cholesterol analogue incorporation for improving in vivo delivery of chemotherapeutics. ACS Appl. Mater. Interfaces.2018;10(6):5213-5226.

13. Wang B, Kuo J, Bae SC, Granick S. When Brownian diffusion is not Gaussian. Nature Mater. 2012;11(6):481-485.

14. Whitehead KA, Langer R, Anderson DG. Knocking down barriers: advances in siRNA delivery. Nat. Rev. Drug Discov.2009;8(2):129-138.

15. Verma A, Stellacci F. Effect of surface properties on nanoparticle-cell interactions. Small. 2010;6(1):1221.

16. Yan Y, Wang Y, Heath JK, Nice EC, Caruso F. Cellular association and cargo release of redox-responsive polymer capsules mediated by exofacial thiols. Adv. Mater. 2011;23(34):3916-3921.

17. Li X, Tang Y-H, Liang H, Karniadakis GE. Large-scale dissipative particle dynamics simulations of self-assembled amphiphilic systems. Chem. Commun. 2014;50(61):8306-8308.

18. Becker OM, Karplus M. Guide to biomolecular simulations. Vol 4: Springer Science \& Business Media; 2006.

19. Gupta R, Badhe Y, Mitragotri S, Rai B. Permeation of nanoparticles across the intestinal lipid membrane: dependence on shape and surface chemistry studied through molecular simulations. Nanoscale.2020;12(11):6318-6333.

20. Zhang Q, Lin JP, Wang LQ, Xu ZW. Theoretical modeling and simulations of self-assembly of copolymers in solution. Prog. Polym. Sci. 2017;75:1-30.

21. Guo XD, Qian Y, Zhang CY, Nie SY, Zhang LJ. Can drug molecules diffuse into the core of micelles? Soft Matter.2012;8(39):9989-9995.

22. Zheng LS, Yang YQ, Guo XD, Sun Y, Qian Y, Zhang LJ. Mesoscopic simulations on the aggregation behavior of pH-responsive polymeric micelles for drug delivery. J. Colloid Interface Sci.2011;363(1):114-121.

23. Xia Q-s, Ding H-m, Ma Y-q. Design strategy of $\mathrm{pH}$-sensitive triblock copolymer micelles for efficient cellular uptake by computer simulations. J. Phys. D: Appl. Phys. 2018;51(12):124002. 
24. Pivkin IV, Karniadakis GE. Accurate coarse-grained modeling of red blood cells. Phys. Rev. Lett. 2008;101(11):118105.

25. Wang S, Guo H, Li Y, Li X. Penetration of nanoparticles across a lipid bilayer: effects of particle stiffness and surface hydrophobicity.Nanoscale. 2019;11(9):4025-4034.

26. Chen L, Li X, Zhang Y, Chen T, Xiao S, Liang H. Morphological and mechanical determinants of cellular uptake of deformable nanoparticles. Nanoscale. 2018;10(25):11969-11979.

27. Zhang S, Gao H, Bao G. Physical principles of nanoparticle cellular endocytosis. ACS Nano. 2015;9(9):8655-8671.

28. Wang W, Yang R, Zhang F, Yuan B, Yang K, Ma Y. Partner-facilitating transmembrane penetration of nanoparticles: a biological test in silico.Nanoscale. 2018;10(24):11670-11678.

29. Huang LY, Yu YS, Lu X, Ding HM, Ma YQ. Designing a nanoparticle-containing polymeric substrate for detecting cancer cells by computer simulations. Nanoscale. 2019;11(5):2170-2178.

30. Liu YC, Li SX, Liu XJ, Sun HN, Yue TT, Zhang XR, Yan B, Cao DP. Design of small nanoparticles decorated with amphiphilic ligands: Self-preservation effect and translocation into a plasma membrane.ACS Appl. Mater. Interfaces. 2019;11(27):23822-23831.

31. Yang K, Ma Y-Q. Computer simulation of the translocation of nanoparticles with different shapes across a lipid bilayer. Nature Nanotech. 2010;5(8):579-583.

32. Groot RD. Electrostatic interactions in dissipative particle dynamics - simulation of polyelectrolytes and anionic surfactants.J. Chem. Phys. 2003;118(24):11265-11277.

33. Liang J, Chen P, Dong B, Huang Z, Zhao K, Yan L-T. Ligand-receptor interaction-mediated transmembrane transport of dendrimer-like soft nanoparticles: Mechanisms and complicated diffusive dynamics.Biomacromolecules. 2016;17(5):1834-1844.

34. Groot RD, Rabone KL. Mesoscopic simulation of cell membrane damage, morphology change and rupture by nonionic surfactants. Biophys. J. 2001;81(2):725-736.

35. Kranenburg M, Smit B. Phase behavior of model lipid bilayers.J. Phys. Chem. B. 2005;109(14):65536563.

36. Shillcock JC, Lipowsky R. Equilibrium structure and lateral stress distribution of amphiphilic bilayers from dissipative particle dynamics simulations. J. Chem. Phys. 2002;117(10):5048-5061.

37. Li X, Gao L, Fang W. Dissipative particle dynamics simulations for phospholipid membranes based on a four-to-one coarse-grained mapping scheme. Plos One. 2016;11(5):0154568.

38. Wan MW, Gao LH, Fang WH. Implicit-solvent dissipative particle dynamics force field based on a four-to-one coarse grained mapping scheme. Plos One. 2018;13(5).

39. Alexeev A, Uspal WE, Balazs AC. Harnessing Janus nanoparticles to create controllable pores in membranes. ACS Nano.2008;2(6):1117-1122.

40. Sun C, Sun J, Xiao G, Zhang H, Qiu X, Li H, Chen L. Mesoscale organization of nearly monodisperse flowerlike ceria microspheres.J. Phys. Chem. B. 2006;110(27):13445-13452.

41. Shillcock JC, Lipowsky R. Tension-induced fusion of bilayer membranes and vesicles. Nature Mater. 2005;4(3):225-228.

42. Wenwen L, Zhao L, Bing Y, Kai Y. Tail-structure regulated phase behaviors of a lipid bilayer. Chinese Physics B.2020;29(12):128701.

43. Wong-Ekkabut J, Baoukina S, Triampo W, Tang IM, Tieleman DP, Monticelli L. Computer simulation study of fullerene translocation through lipid membranes. Nature Nanotech. 2008;3(6):363-368. 
44. Gupta R, Rai B. Effect of size and surface charge of gold nanoparticles on their skin permeability: A molecular dynamics study.Sci. Rep. 2017;7(1):45292.

45. Dallavalle M, Calvaresi M, Bottoni A, Melle-Franco M, Zerbetto F. Graphene can wreak havoc with cell membranes. ACS Appl. Mater. Interfaces. 2015;7(7):4406-4414.

46. Mao J, Guo R, Yan L-T. Simulation and analysis of cellular internalization pathways and membrane perturbation for graphene nanosheets. Biomaterials. 2014;35(23):6069-6077.

47. Gao H, Shi W, Freund LB. Mechanics of receptor-mediated endocytosis.Proceedings of the National Academy of Sciences of the United States of America. 2005;102(27):9469-9474.

48. Deserno M. Elastic deformation of a fluid membrane upon colloid binding. Phys. Rev. E. 2004;69(3):031903.

49. Ding H-m, Ma Y-q. Interactions between Janus particles and membranes. Nanoscale. 2012;4(4):11161122 .

50. Gao X, Dong J, Zhang X. The effect of nanoparticle size on endocytosis dynamics depends on membranenanoparticle interaction. Mol. Simulat. 2015;41(7):531-537.

51. Orsi M, Haubertin DY, Sanderson WE, Essex JW. A quantitative coarse-grain model for lipid bilayers. J. Phys. Chem. B.2008;112(3):802-815.

52. Huang C, Zhang Y, Yuan H, Gao H, Zhang S. Role of nanoparticle geometry in endocytosis: Laying down to stand up. Nano Lett.2013;13(9):4546-4550.

53. Chen P, Yue H, Zhai X, Huang Z, Ma G-H, Wei W, Yan L-T. Transport of a graphene nanosheet sandwiched inside cell membranes. Sci. Adv.2019;5(6):eaaw3192.

54. Xu Z, Gao L, Chen P, Yan L-T. Diffusive transport of nanoscale objects through cell membranes: a computational perspective. Soft Matter. 2020;16(16):3869-3881.

55. Gu Y, Sun W, Wang G, Zimmermann MT, Jernigan RL, Fang N. Revealing rotational modes of functionalized gold nanorods on live cell membranes.Small. 2013;9(5):785-792.

56. Li SX, Luo Z, Xu Y, Ren H, Deng L, Zhang XR, Huang F, Yue TT. Interaction pathways between soft lipid nanodiscs and plasma membranes: A molecular modeling study. Biochim. Biophys. Acta, Biomembr.2017;1859(10):2096-2105.

57. Lv K, Li Y. Indentation of graphene-covered atomic force microscopy probe across a lipid bilayer membrane: Effect of tip shape, size, and surface hydrophobicity. Langmuir. 2018;34(26):7681-7689.

58. Yi X, Shi X, Gao H. A universal law for cell uptake of one-dimensional nanomaterials. Nano Lett. 2014;14(2):1049-1055.

59. Li Y, Kroger M, Liu WK. Shape effect in cellular uptake of PEGylated nanoparticles: comparison between sphere, rod, cube and disk. Nanoscale. 2015;7(40):16631-16646.

60. Chen P, Huang Z, Liang J, Cui T, Zhang X, Miao B, Yan L-T. Diffusion and directionality of charged nanoparticles on lipid bilayer membrane.ACS Nano. 2016;10(12):11541-11547.

61. Liu B, Goree J. Superdiffusion and non-gaussian statistics in a driven-dissipative 2D dusty plasma. Phys. Rev. Lett.2008;100(5):055003.

62. Baskaran A, Marchetti MC. Enhanced diffusion and ordering of self-propelled rods. Phys. Rev. Lett. 2008;101(26):268101.

63. Ji Q-J, Yuan B, Lu X-M, Yang K, Ma Y-Q. Controlling the nanoscale rotational behaviors of nanoparticles on the cell membranes: A computational model. Small. 2016;12(9):1140-1146. 
64. Zhang LY, Zhao YP, Wang XQ. Nanoparticle-mediated mechanical destruction of cell membranes: A coarse-grained molecular dynamics study. ACS Appl. Mater. Interfaces. 2017;9(32):26665-26673.

65. Li Y, Chen X, Gu N. Computational investigation of interaction between nanoparticles and membranes: Hydrophobic/hydrophilic effect.J. Phys. Chem. B. 2008;112(51):16647-16653.

66. Liang Q. Penetration of polymer-grafted nanoparticles through a lipid bilayer. Soft Matter. 2013;9(23):5594-5601.

67. Zhang HZ, Ji QJ, Huang CJ, Zhang SL, Yuan B, Yang K, Ma YQ. Cooperative transmembrane penetration of nanoparticles. Sci. Rep.2015;5:10525.

68. Chen P, Yan L-T. Physical principles of graphene cellular interactions: computational and theoretical accounts. J. Mater. Chem. B. 2017;5(23):4290-4306.

69. Pogodin S, Slater NKH, Baulin VA. Surface patterning of carbon nanotubes can enhance their penetration through a phospholipid bilayer.ACS Nano. 2011;5(2):1141-1146.

70. Lin $\mathrm{X}, \mathrm{Gu} \mathrm{N}$. Surface properties of encapsulating hydrophobic nanoparticles regulate the main phase transition temperature of lipid bilayers: A simulation study. Nano Res. 2014;7(8):1195-1204.

71. Ding H-m, Ma Y-q. Controlling cellular uptake of nanoparticles with pH-sensitive polymers. Sci. Rep. 2013;3(1):2804.

72. Hao LX, Lin L, Zhou J. pH-responsive zwitterionic copolymer dha-pblg-pcb for targeted drug delivery: A computer simulation study.Langmuir. 2019;35(5):1944-1953.

73. Shen ZQ, Ye HL, Kroger M, Li Y. Aggregation of polyethylene glycol polymers suppresses receptormediated endocytosis of PEGylated liposomes. Nanoscale. 2018;10(9):4545-4560.

74. Hu J-m, Tian W-d, Ma Y-q. Computational investigations of arginine-rich peptides interacting with lipid membranes. Macromol. Theory Simul. 2015;24(4):399-406.

75. Albanese A, Chan WCW. Effect of gold nanoparticle aggregation on cell uptake and toxicity. ACS Nano. 2011;5(7):5478-5489.

76. Li Y, Yuan B, Yang K, Zhang X, Yan B, Cao D. Counterintuitive cooperative endocytosis of likecharged nanoparticles in cellular internalization: computer simulation and experiment.Nanotechnology. 2017;28(8):085102.

77. Ni S-d, Yin Y-w, Li X-l, Ding H-m, Ma Y-q. Controlling the interaction of nanoparticles with cell membranes by the polymeric tether. Langmuir. 2019;35(39):12851-12857.

78. Wang S, Li X, Gong X, Liang H. Mechanistic modeling of spontaneous penetration of carbon nanocones into membrane vesicles. Nanoscale. 2020;12(4):2686-2694.

79. Yi X, Shi X, Gao H. Cellular uptake of elastic nanoparticles.Phys. Rev. Lett. 2011;107(9):098101.

80. Li Y, Feng DW, Zhang XR, Cao DP. Design strategy of cell-penetrating copolymers for high efficient drug delivery. Biomaterials.2015;52:171-179.

81. Li JW, Wang JF, Yao Q, Yu K, Yan YG, Zhang J. Cooperative assembly of Janus particles and amphiphilic oligomers: the role of Janus balance.Nanoscale. 2019;11(15):7221-7228.

82. Guo XD, Zhang LJ, Qian Y. Systematic multiscale method for studying the structure-performance relationship of drug-delivery systems.Ind. Eng. Chem. Res. 2012;51(12):4719-4730.

83. Xia QS, Ding HM, Ma YQ. Can dual-ligand targeting enhance cellular uptake of nanoparticles? Nanoscale. 2017;9(26):8982-8989. 
84. Guo XD, Zhang LJ, Wu ZM, Qian Y. Dissipative particle dynamics studies on microstructure of $\mathrm{pH}-$ sensitive micelles for sustained drug delivery. Macromolecules. 2010;43(18):7839-7844.

Figure. 1. Main content of this review. (Some details of this figure are reused from Reference ${ }^{81}$ with permission from The Royal Society of Chemistry, Reference ${ }^{82}$ with permission from Copyright (C) 2012, American Chemical Society, Reference ${ }^{33}$ with permission from Copyright (C) 2016, American Chemical Society, Reference $^{26}$ with permission from The Royal Society of Chemistry, Reference ${ }^{83}$ with permission from The Royal Society of Chemistry, Reference ${ }^{28}$ with permission from The Royal Society of Chemistry, Reference ${ }^{64}$ with permission from Copyright (C) 2017, American Chemical Society.)

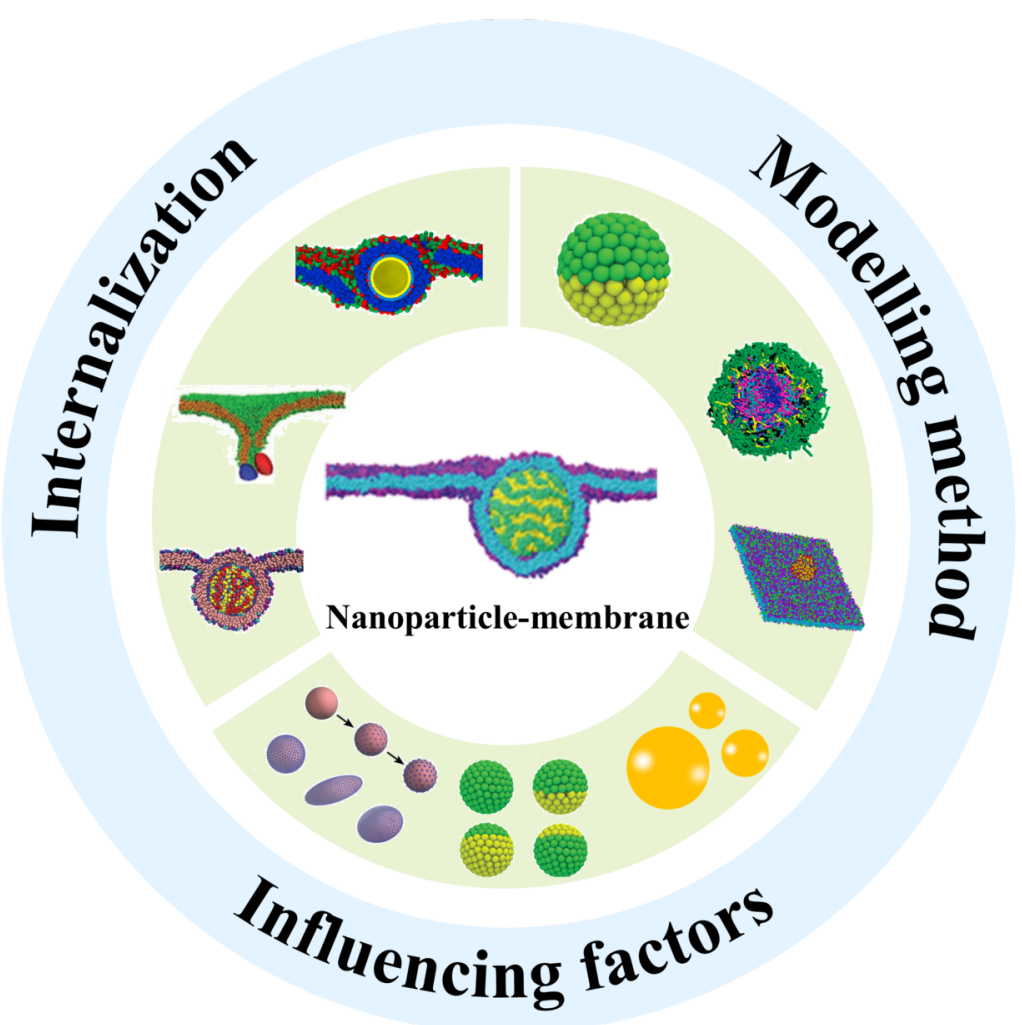

Figure. 2. Several typical nanoparticle models. A. Rigid nanoparticles, reproduced from Reference ${ }^{81}$ with permission from The Royal Society of Chemistry. B. Soft matter nanoparticles, reproduced from Reference ${ }^{84}$ with permission from Copyright (C) 2010, American Chemical Society. C. Nanoparticles with controllable elastic modulus, reproduced from Reference ${ }^{26}$ with permission from The Royal Society of Chemistry. 


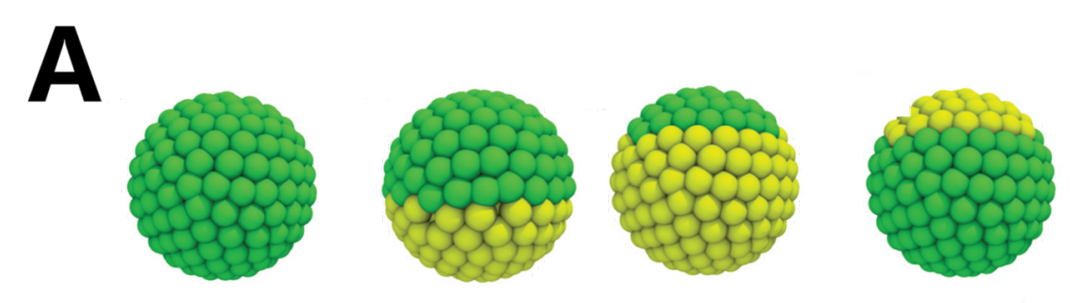

B
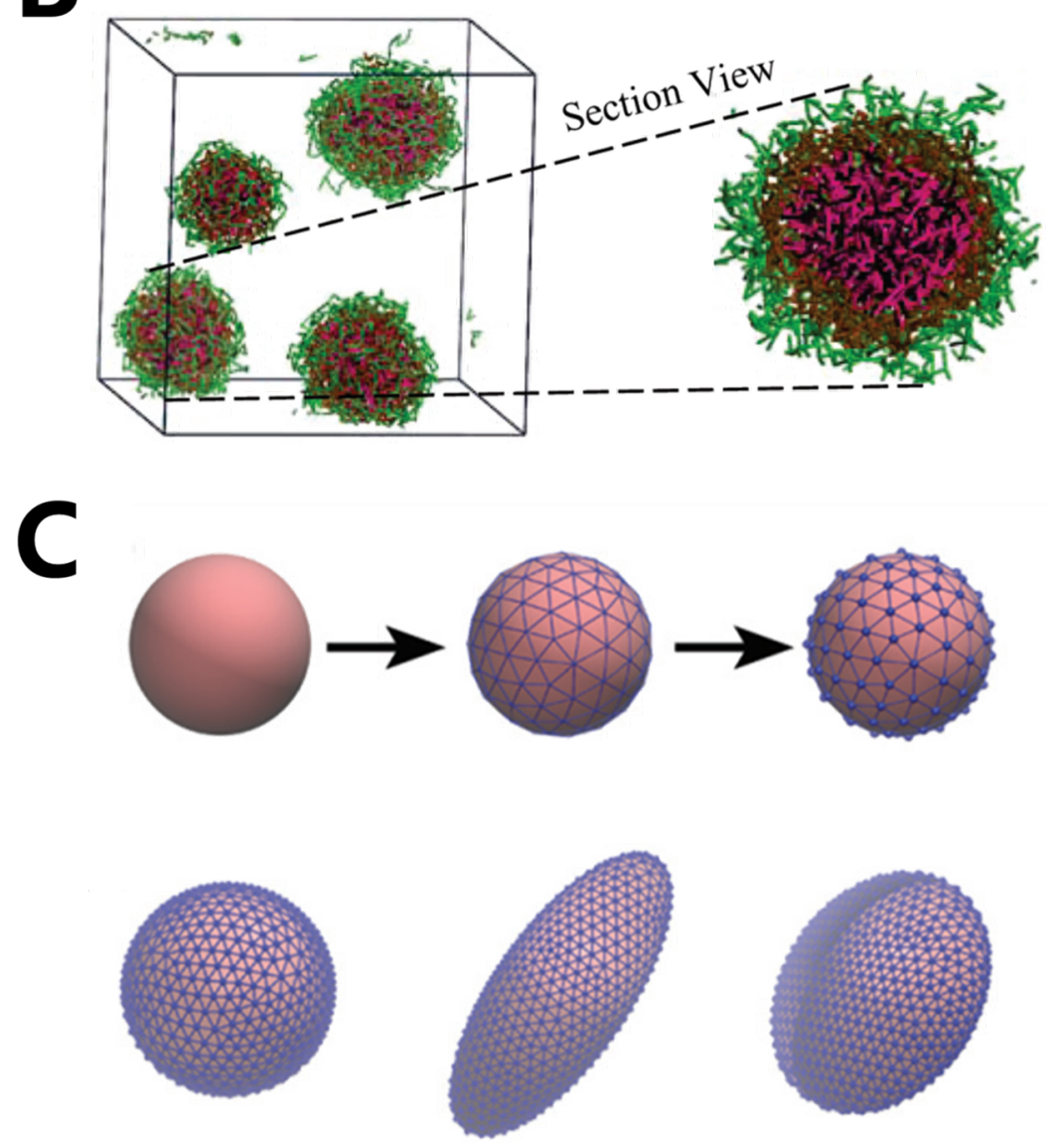

Figure. 3. Several typical nanoparticle models. A. Commonly used phospholipid coarse-grained model, reproduced from Reference ${ }^{37}$ with permission from (C) $2016 \mathrm{Li}$ et al.. B. Single-component phospholipid bimolecular membrane model, reproduced from Reference ${ }^{33}$ with permission from Copyright (C) 2016, American Chemical Society. C. Multi-component phospholipid bimolecular membrane model, reproduced from Reference $^{19}$ with permission from The Royal Society of Chemistry. 


\section{A}
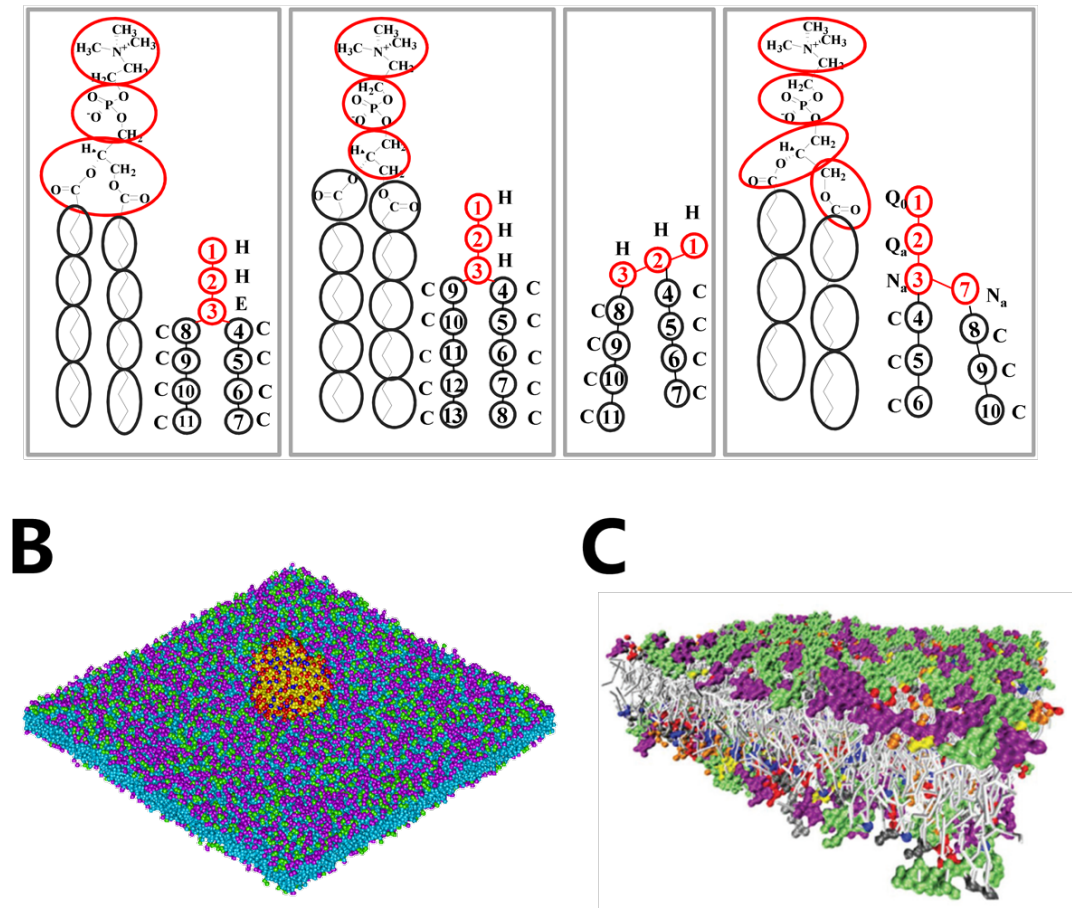

Figure. 4. The size effect of nanoparticles: A. Large-size nanoparticles will cause more significant cell membrane deformation, reproduced from Reference ${ }^{44}$ with permission from Copyright (C) 2016, Springer Nature. B. As the size is smaller than the optimal size, large-size nanoparticles are more likely to be wrapped by cell membranes than small-size particles, reproduced from Reference ${ }^{50}$ with permission from Copyright (C) 2014, Taylor \& Francis. 

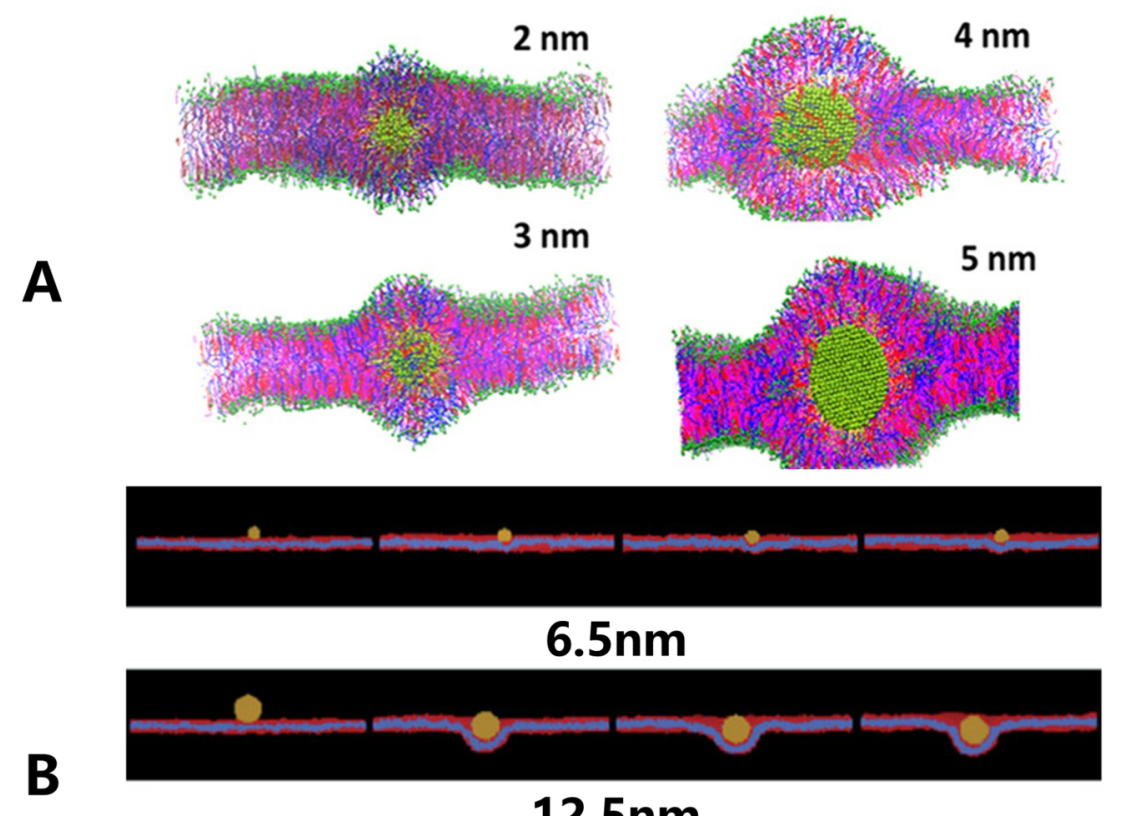

$12.5 \mathrm{~nm}$

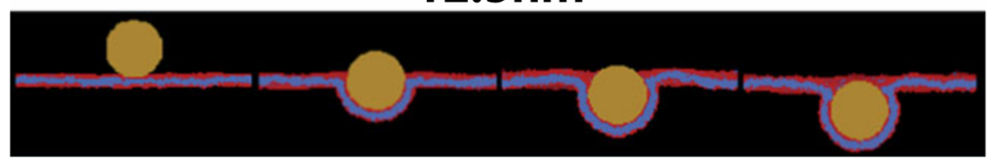

$25 \mathrm{~nm}$

Figure. 5. The shape effect of nanoparticles: A. Orientation of anisotropic nanoparticles increases membrane curvature. B. The particular dynamic process of anisotropic materials: orientation-wrappingreorientation, reproduced from Reference ${ }^{19}$ with permission from The Royal Society of Chemistry. C. Comparison of the endocytosis efficiency of isotropic nanoparticles and that of anisotropic nanoparticles, reproduced from Reference ${ }^{59}$ with permission from The Royal Society of Chemistry. D. The process in which deformable spherical nanoparticles are wrapped by cell membranes and deform into asymmetrically shape, reproduced from Reference ${ }^{26}$ with permission from The Royal Society of Chemistry. 

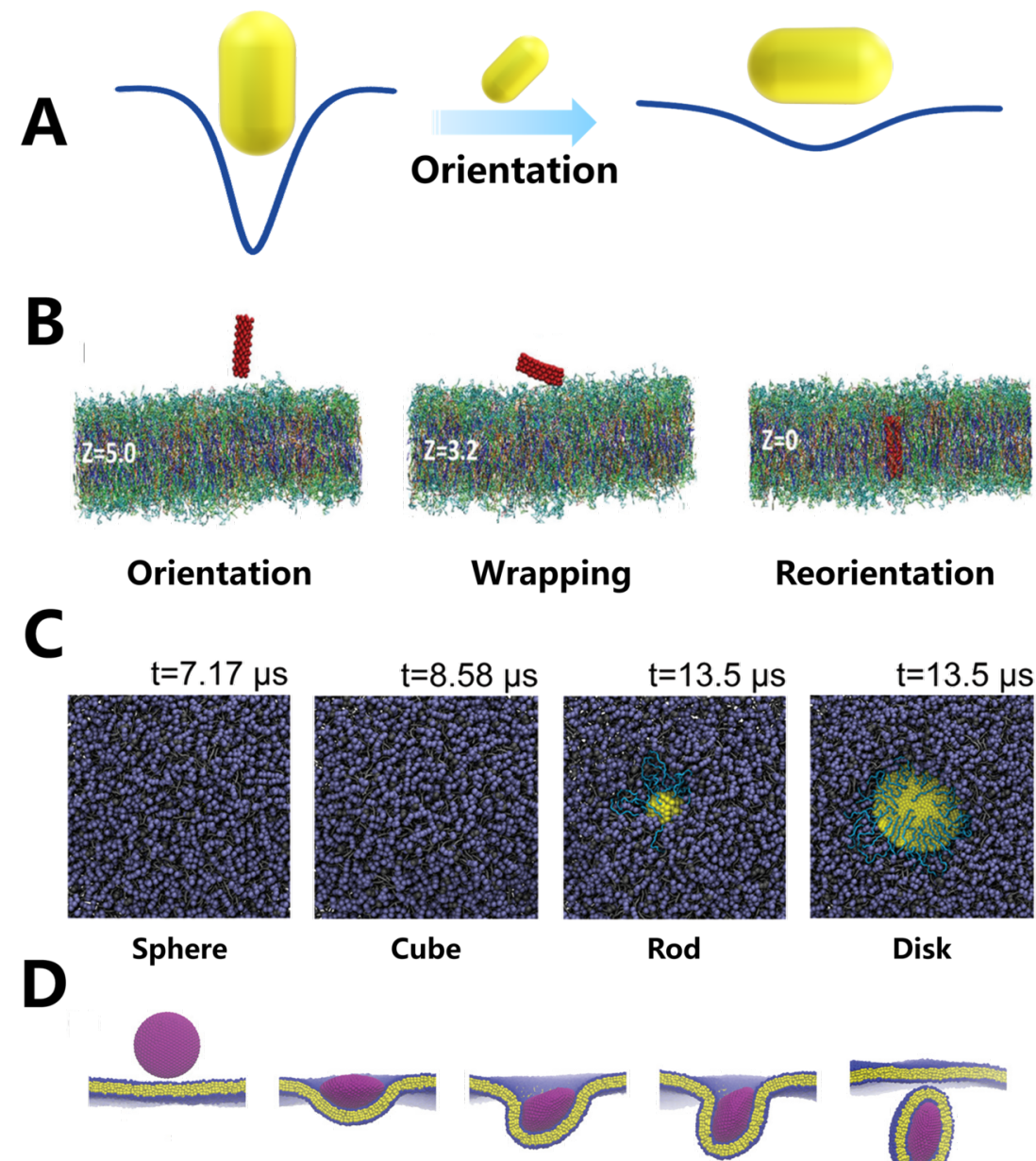

$\begin{array}{lll}\text { (i) } 0 \tau & \text { (ii) } 2500 \tau & \text { (iii) } 6500 \tau\end{array}$

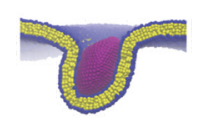

(iv) $7750 \tau$

Reorientation

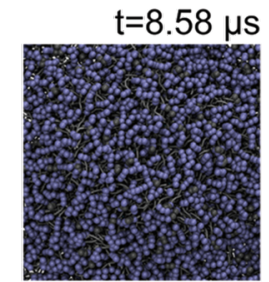

Cube



Rod

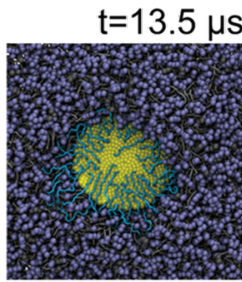

Disk

Figure. 6. The surface property effect of nanoparticles: A. Hydrophilic / hydrophobic surface distribution affects the penetration efficiency, reproduced from Reference ${ }^{67}$ with permission from Copyright (C) 2016, Springer Nature. B. The surface charge property of nanoparticles affects the cell membrane deformation, reproduced from Reference ${ }^{60}$ with permission from Copyright (C) 2016, American Chemical Society. C. The relationship between nanoparticle ligand density and cell membrane tension, reproduced from Reference ${ }^{33}$ with permission from Copyright (C) 2016, American Chemical Society. 

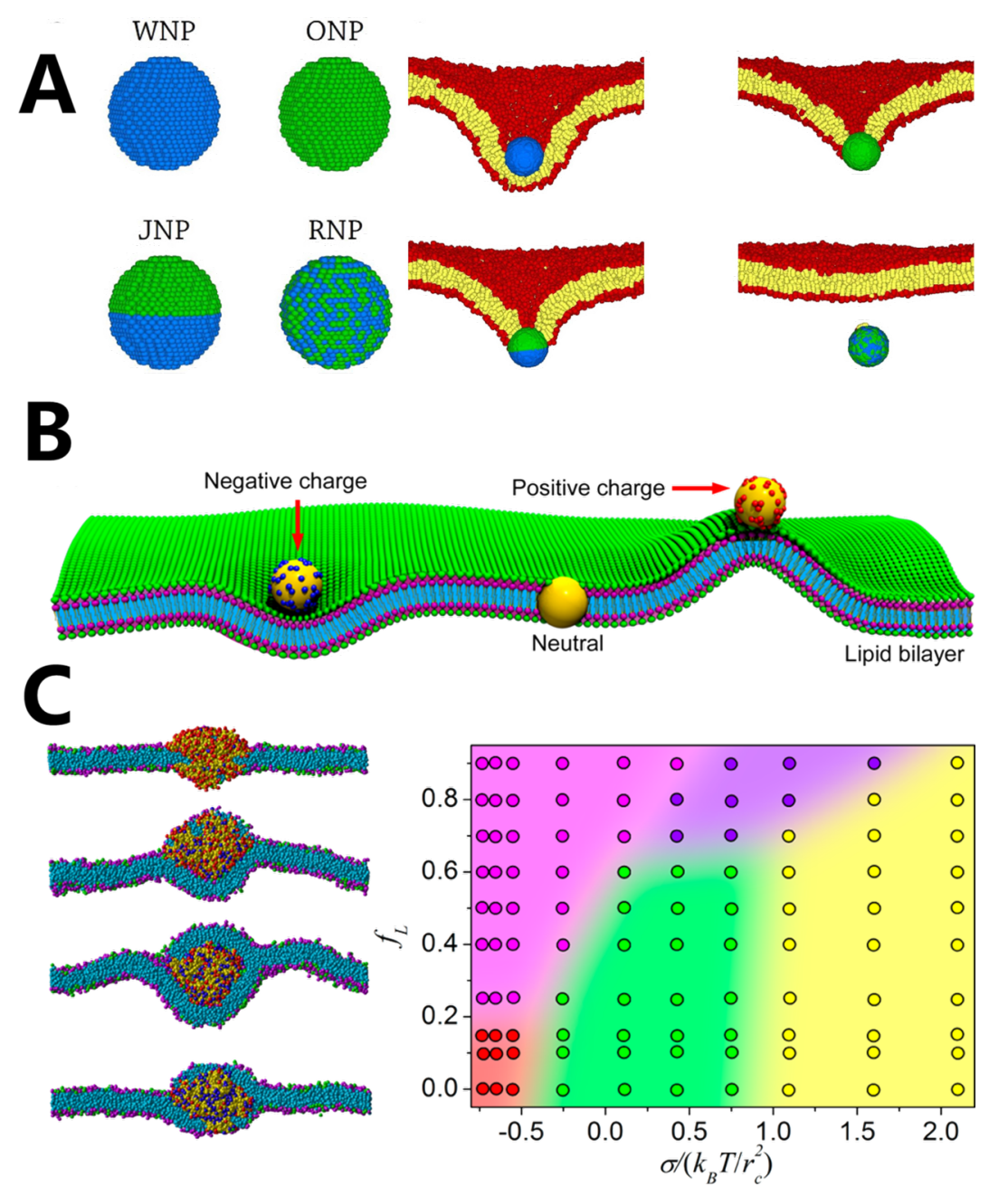

Figure. 7. The concentration effect of nanoparticles: A. Cooperative nanoparticle internalization effect of nanoparticles with different surface properties, reproduced from Reference ${ }^{67}$ with permission from The Royal Society of Chemistry. B. The effect of properties of dual-polymer-tether linked nanoparticles on the degree of cell membrane encapsulation, reproduced from Reference ${ }^{77}$ with permission from Copyright (C) 2019, American Chemical Society. 


\section{A Hydrophilic \\ Nanoparticle}

Randomly coated

Nanoparticle
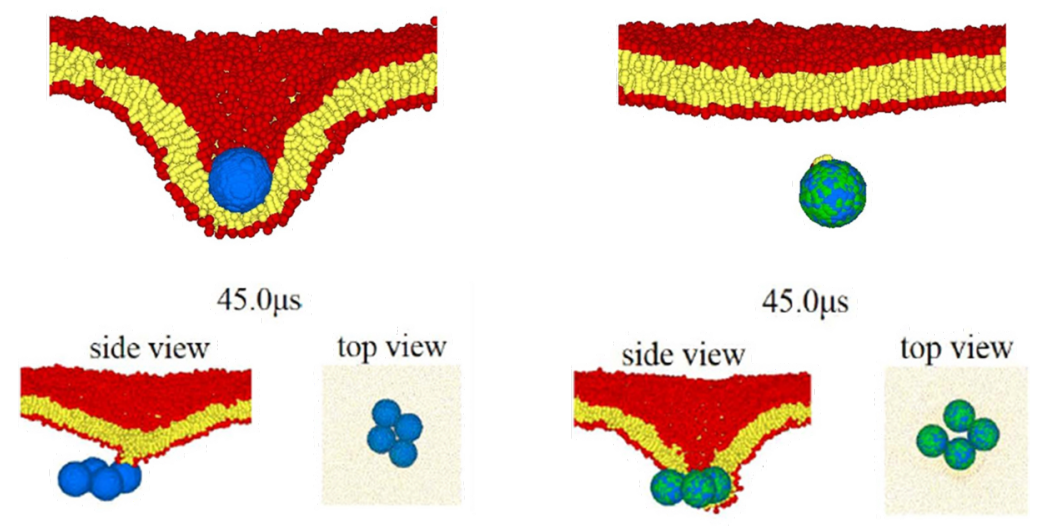

B
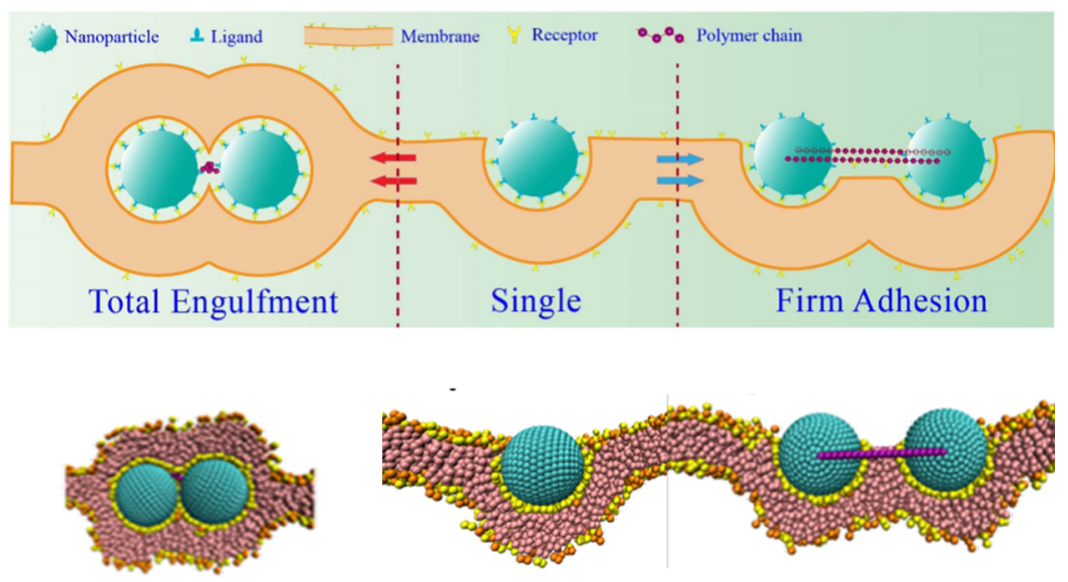

Figure. 8. The mechanical property effect of nanoparticles: A. Effect of elastic modulus on the internalization of hydrophilic / hydrophobic nanoparticles, reproduced from Reference ${ }^{25}$ with permission from The Royal Society of Chemistry. B. Effect of elastic modulus on the cell internalization efficiency of nanoparticles with various shapes, reproduced from Reference ${ }^{26}$ with permission from The Royal Society of Chemistry. 
A

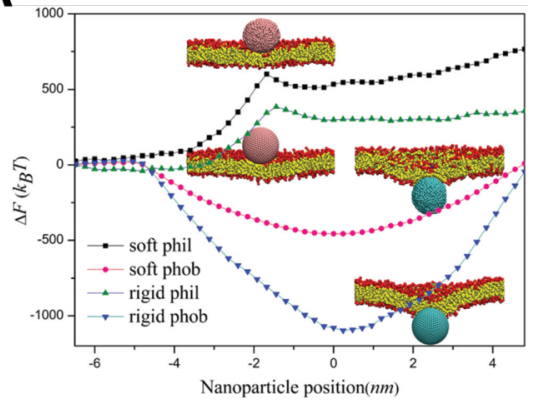

B

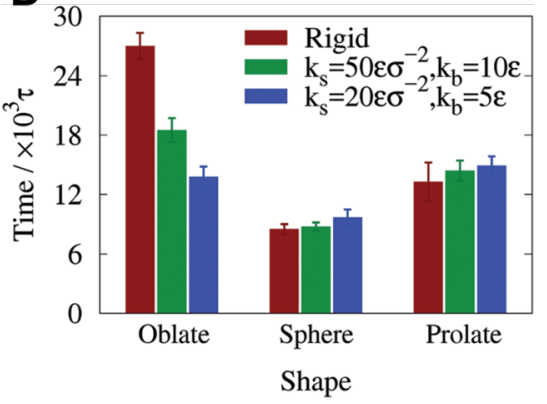

